Rationalisierung im Konsum 

Dirk Dalichau

Rationalisierung im Konsum

Eine ethnographische Studie von

Einkaufspraktiken am Beispiel von

Frankfurt am Main

Springer VS 
Dirk Dalichau

Frankfurt am Main, Deutschland

Dissertation an der Goethe-Universität Frankfurt, 2014

D 30

ISBN 978-3-658-13129-6

ISBN 978-3-658-13130-2 (eBook)

DOI 10.1007/978-3-658-13130-2

Die Deutsche Nationalbibliothek verzeichnet diese Publikation in der Deutschen Nationalbibliografie; detaillierte bibliografi sche Daten sind im Internet über http://dnb.d-nb.de abrufbar.

\section{Springer VS}

(C) Springer Fachmedien Wiesbaden 2016

Das Werk einschließlich aller seiner Teile ist urheberrechtlich geschützt. Jede Verwertung, die nicht ausdrücklich vom Urheberrechtsgesetz zugelassen ist, bedarf der vorherigen Zustimmung des Verlags. Das gilt insbesondere für Vervielfältigungen, Bearbeitungen, Übersetzungen, Mikroverfilmungen und die Einspeicherung und Verarbeitung in elektronischen Systemen.

Die Wiedergabe von Gebrauchsnamen, Handelsnamen, Warenbezeichnungen usw. in diesem Werk berechtigt auch ohne besondere Kennzeichnung nicht zu der Annahme, dass solche Namen im Sinne der Warenzeichen- und Markenschutz-Gesetzgebung als frei zu betrachten wären und daher von jedermann benutzt werden dürften.

Der Verlag, die Autoren und die Herausgeber gehen davon aus, dass die Angaben und Informationen in diesem Werk zum Zeitpunkt der Veröffentlichung vollständig und korrekt sind. Weder der Verlag noch die Autoren oder die Herausgeber übernehmen, ausdrücklich oder implizit, Gewähr für den Inhalt des Werkes, etwaige Fehler oder Äußerungen.

Gedruckt auf säurefreiem und chlorfrei gebleichtem Papier

Springer VS ist Teil von Springer Nature

Die eingetragene Gesellschaft ist Springer Fachmedien Wiesbaden GmbH 


\section{Inhaltsverzeichnis}

Inhaltsverzeichnis ....................................................

Verzeichnis der Abbildungen.................................................................... ix

Verzeichnis der Übersichten ....................................................................ix

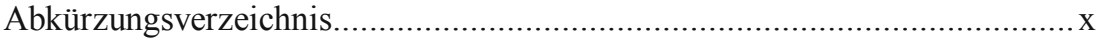

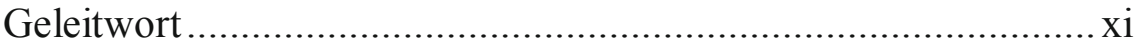

Vorwort .................................................................xiii

1. Einleitung........................................................... 1

2. Konsum - Theoretische Perspektive.................................... 7

2.1. Konsum. Verortung im Forschungsfeld ............................................. 8

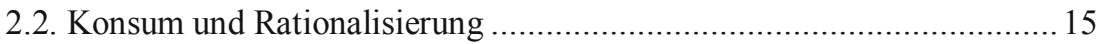

2.2.1. Konsum auf dem Weg zur Rationalisierung.......................... 15

2.2.2. Rationalisierter Konsum aus der Theorieperspektive ............. 22

2.2.3. Rationalisierter Konsum: Gegen- und Folgeentwicklungen ..... 32

2.3. Konsum und Individuum......................................................................... 41

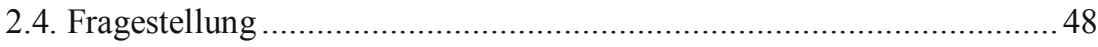

3. Soziale (Konsum-) Praktiken.............................................. 53

3.1. Soziale Praktiken: Begriff .................................................................5

3.2. Soziale Praktiken: Theorie(n) …………........................................ 56

3.3. Soziale Praktiken und Konsum........................................................ 77

3.4. Implikationen für das weitere Vorgehen ........................................... 82

4. Methode und Vorgehen ............................................... 87

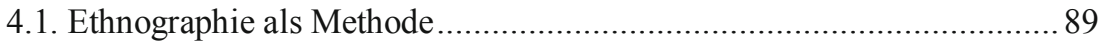


4.2. Soziologische Ethnographie ........................................................ 96

4.2.1. Grundverständnis soziologischer Ethnographie ..................... 96

4.2.2. Varianten der (soziologischen) Ethnographie ...................... 100

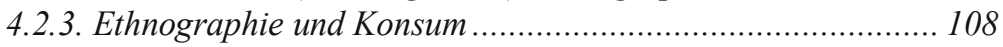

4.2.4. Zusammenfassung: Ethnographie in dieser Studie ............... 109

4.3. Ethnographische Erhebungsinstrumente ........................................... 110

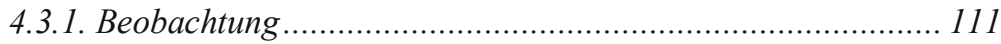

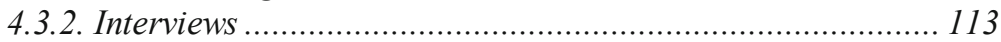

4.3.3. Visuelle Ethnographie .................................................... 116

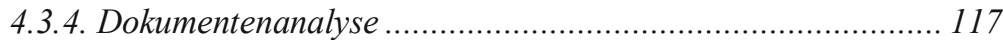

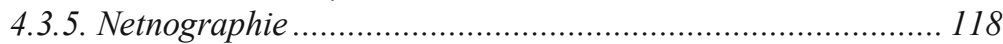

4.3.6. Weiteres Material............................................................... 119

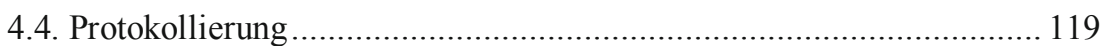

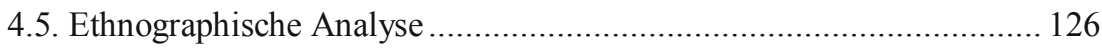

4.6. Ethnographische Typologie......................................................... 129

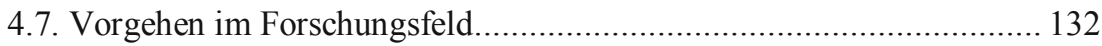

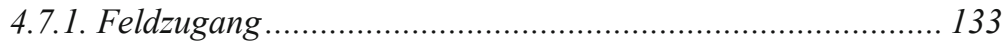

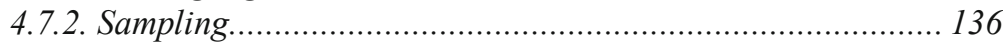

4.7.3. Verhalten im Feld ...................................................... 138

4.7.4. Dauer der Feldphase.................................................... 140

5. Praxisfeld Einzelhandel .............................................. 143

5.1. Bedingungen an das Praxisfeld.......................................................... 144

5.2. Auswahl des Praxisfeldes Frankfurt..................................................... 146

5.3. Vorstellung des Praxisfeldes Frankfurt ............................................... 150

5.3.1. Praxisfeld Frankfurter Einzelhandel im Bezug zur Stadt ...... 150

5.3.2. Praxisfeld Frankfurter Einzelhandel im Detail.................... 152

6. Ethnographien der Einkaufsstraßen ..................................... 159

6.1. Ethnographie der Schweizer Straße ................................................ 160

6.2. Ethnographie der Leipziger Straße .................................................... 169

6.3. Ethnographie der Berger Straße..................................................... 178

6.4. Ethnographie der Zeil..................................................................... 185 
7.1. Einkaufspraktiken in der Einzelbetrachtung....................................... 194

7.1.1. Sequenz: Betreten.......................................................... 195

7.1.2. Sequenz: Auswahl und Bestellung.................................... 204

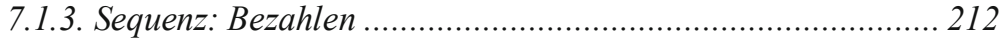

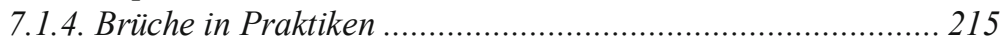

7.1.5. Brüche in Sequenzen .................................................... 217

7.2. Einkaufspraktiken und ihre Variationen.......................................... 218

7.2.1. Varianten der Basispraktiken - Einflussfaktoren .................. 219

7.2.2. Varianten der Basispraktiken - Differenzierungsfaktoren ...... 223

7.2.3. Filialisierung - der mittelbare Einfluss .............................. 228

7.2.4. Filialisierung - der unmittelbare Einfluss ........................... 233

7.3. Zentrale Erkenntnisse aus den Kurzethnographien ............................ 237

7.4. Gesamtbetrachtung als Zusammenführung der Teilergebnisse............. 241

7.5. Rückbezug zum theoretischen Orientierungsrahmen .......................... 245

8. Resümee .................................................................. 249

Literaturverzeichnis .................................................... 257 



\section{Verzeichnis der Abbildungen}

Abb. 1: Entwicklung der Marktanteile verschiedener Betriebstypen ................ 21

Abb. 2: Anteil der Einkaufstypen in deutschen Innenstädten nach Schuckel..... 45

Abb. 3: Prozess der Konsumveränderungen..................................................... 49

Abb. 4: Fokussierte und konventionelle Ethnographie im Vergleich nach

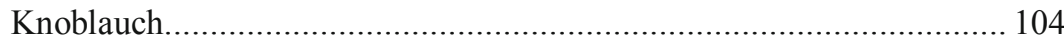

Abb. 5: Erfassungsebenen der Feldnotizen ................................................. 123

Abb. 6: Sechs Analyseschritte nach Hammersley und Atkinson. .................... 128

Abb. 7: Räumliche Bezugsebenen von Konsumpraktiken ............................. 146

Abb. 8: Filialisierung in den großen Einkaufsstraßen in Frankfurt am Main ... 149

Abb. 9: Auswirkung des Bedienkonzepts auf die endogenen

Differenzierungsfaktoren nach Distributionstyp (filialisiert versus

inhabergeführt) unter Angabe der Fallzahlen (Geschäfte) ...................... 234

Abb. 10: Auswirkung der Ladengröße auf die endogenen

Differenzierungsfaktoren nach Distributionstyp (filialisiert versus inhabergeführt) unter Angabe der Fallzahlen (Geschäfte) ...................... 236

Abb. 11: Übersicht der Einkaufspraktiken und der Einflussgrößen ................. 243

\section{Verzeichnis der Übersichten}

Übersicht 1: Phasen des Konsumwandels in Deutschland ab 1940 nach

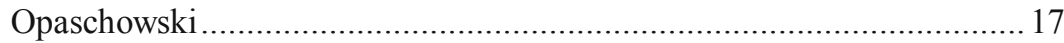

Übersicht 2: Konsumtypen nach Opaschowski .............................................. 44

Übersicht 3: Konsumententypen: Erlebniskäufer?...........................................47

Übersicht 4: Drei Bescheidenheiten zum Verhalten im Feld nach Dellwing und

Prus 


\section{Abkürzungsverzeichnis}

\section{Anm. Anmerkung}

ANT Akteur-Netzwerk-Theorie / Actor-Network Theory

bzw. beziehungsweise

etc. Et cetera

o.A. ohne Autor

o.J. ohne Jahr

o.S. ohne Seite 


\section{Geleitwort}

Vor einiger Zeit lief ich durch die Leipziger Straße in Frankfurt und fand mich plötzlich vor der kleinen Kaffeerösterei wieder, von der Dirk Dalichau im Rahmen eines Vortrags im Forschungskolloquium erzählt hatte. Ich muss gestehen, ich bin nicht hineingegangen, vermutlich, weil ich keine Lust auf intensive soziale Interaktionen hatte, wie sie Dalichau als typisch für den Eigentümer geführten Einzelhandel beschrieben hat. Kaffee, so dachte ich, kann man auch in der anonymen, auf Selbstbedienung basierenden Filiale eines großen Einzelhandelsunternehmens kaufen.

Wir antizipieren also, wenn wir die Entscheidung treffen, ein Geschäft zu betreten, was uns dort erwartet, und passen unser Verhalten den dort vorgefundenen Rahmenbedingungen - mehr oder weniger gut - an.

Dirk Dalichau nimmt die historisch zu beobachtende Filialisierung des Einzelhandels zum Ausgangspunkt seiner Überlegungen. Es geht ihm um die Frage, inwieweit wir unser Einkaufshandeln je nach Kontext variieren. Dafür hat er vier Einkaufsstraßen in Frankfurt, die sich im Hinblick auf die Zusammensetzung von Geschäften nach Größe und Bedienmodell unterschieden, einer ethnographischen Untersuchung unterzogen. Konzeptionell geht er davon aus, dass wir es beim Einkaufen mit einer komplexen sozialen Praktik zu tun haben, die sich durch Routine und spezifische Kenntnisse und Kompetenzen auszeichnet. So ist unsere Einkaufsroutine in kleinen, bedienten Läden eine andere als in großen Selbstbedienungsläden. Dabei vermögen wir es beim Einkaufen, von einem Kontext zum anderen zu wechseln. Dieses Phänomen ist nicht trivial, wenn man die Erkenntnis von Dalichau berücksichtigt, wonach das Einkaufshandeln ein komplexes Unterfangen ist, was zumindest aus vier Phasen besteht: Eintreten in einen Laden, Orientierung im Laden und Auswahl von Gütern und Bezahlen. Die unterschiedlichen Bedienmodelle erfordern je unterschiedliche Praktiken.

Die vorliegende Arbeit, die man im Übrigen auch von hinten - Empirie nach vorne lesen kann, besticht durch die Genauigkeit und Tiefe der Analyse des Einkaufsverhaltens in unterschiedlichen Kontexten. Sie leistet darüber hinaus eine wichtige Grundlage für weitere Studien in diesem Feld. Dalichau verweist 
am Ende selbst auf mögliche weitergehende und vielversprechende Fragestellungen, so z.B., inwieweit wir durch die zunehmende Filialisierung des Einzelhandels spezifische - vor allem soziale - Kompetenzen verlieren werden, oder, was genau uns bewegt, an bestimmten Tagen, spezifische Bedienkonzepte alternativen Angeboten vorzuziehen.

Prof. Dr. Birgit Blättel-Mink, Frankfurt im November 2015 


\section{Vorwort}

Im Zuge dieser Arbeit konnte ich die Stadt Frankfurt am Main aus einer ganz neuen Perspektive kennenlernen. Ich bin in der Region Rhein-Main aufgewachsen, habe in Frankfurt am Main studiert und lebe hier. Die Gespräche mit den Menschen in den Geschäften und das Beobachten in den Straßenzügen und kleinen und großen Läden führten zu einem neuen Blick auf diese Stadt und ihr Einzelhandelsangebot. Ich möchte an dieser Stelle allen danken, deren Konsumund Einkaufshandlungen zum Erkenntnisgewinn in dieser Arbeit führten. Ich wünsche dem bestehenden vielseitigen Angebot in Frankfurts Einkaufsstraßen noch ein langes Bestehen, steten Wandel auf Augenhöhe der Zeit, gemischt mit resistentem Fortbestehen liebgewonnener Angebote, auch wenn diese vielleicht nicht mehr ganz in die aktuelle Zeit passen mögen. Es ist der Mix, der noch vorhanden ist, und der den Charme so manches Straßenzugs ausmacht.

Diese Arbeit und ihre Entstehungsgeschichte wurden von einigen Personen begleitet, von denen kontroverse Diskussionen, angeregter Meinungsaustausch, aufbauende Worte, gemeinsame Konsumerlebnisse, interessante Gedanken und nicht zuletzt auch ausgleichende Freizeitaktivitäten ausgingen. So manche Diskussion zum Thema rief schlaflose Nächte hervor, bis die Gedanken dann auf dem nun vorliegenden Wege letztlich Eingang in die Arbeit nehmen konnten. Für die intensive und langjährige Betreuung gilt Birgit Blättel-Mink ein ganz besonderer Dank - auch dafür, dass es immer meine Arbeit bleiben konnte, die über die vielen wertvollen Hinweise und Gespräche von ihr sehr unterstützt wurde. Ebenso gilt mein Dank auch Tilla Siegel, insbesondere für ihre Rationalisierungshinweise, sowohl aus soziologischer Sicht als auch den Schreibprozess betreffend. Die Diskussionen in unserem gemeinsamen Kolloquium waren manchmal verwirrend, meistens engagiert, aber immer gewinnbringend. Ich danke Euch beiden dafür!

Aus den verschiedensten Gründen und für die teilweise unendliche Geduld, wenn ich mal wieder kein anderes Thema außer Einkaufspraktiken mehr kannte, danke ich außerdem meinem gesamten Umfeld, insbesondere Anne B., meinem langjährigen Italienischkurs, Christina, Marco, Jens, Lukas, Ruth, Vera, Johanna, Anne L., Renate, Daniel, Raphael, Gisela, Gerhard, Dan, Saskia und Frauke. Von nächtelangen Diskussionen bis zu ausgleichenden Skiurlauben, von umfas- 
sender Kritik bis zu jahrelanger Geduld, von ungeordneten Ideen bis zur letzten Formatierung, bei wochenlangen Fußmärschen über die Alpen wie auch bei einem gemütlichen Ebbelwoi, während zahlreicher Aufenthalte in den Einkaufsstraßen und bei vielen Treffen des Kolloquiums, bei gemeinsamen Mittagessen in der Mensa ebenso wie durch das Führen grundsätzlicher Konsumdebatten und durch zahlreiche Hinweise von der ersten Idee bis zur Publikation habt Ihr alle diese Arbeit irgendwie, mal mehr, mal weniger begleitet - ich danke Euch allen dafür! 